Łukasz Delong (Warszawa)

\title{
EXPONENTIAL UTILITY OPTIMIZATION, INDIFFERENCE PRICING AND HEDGING FOR A PAYMENT PROCESS
}

Abstract. We deal with pricing and hedging for a payment process. We investigate a Black-Scholes financial market with stochastic coefficients and a stream of liabilities with claims occurring at random times, continuously over the duration of the contract and at the terminal time. The random times of the claims are generated by a random measure with a stochastic intensity of jumps. The claims are written on the asset traded in the financial market and on the non-tradeable source of risk driven by the random measure. Our framework allows us to consider very general streams of liabilities which may arise in financial and insurance applications. We solve the exponential utility optimization problem for our payment process and we derive the indifference price and hedging strategy. We apply backward stochastic differential equations.

1. Introduction. Optimal investment problems have been extensively studied in the literature since Harry Markowitz introduced the problem of mean-variance portfolio selection (see Markowitz (1952)), and Robert Merton found the optimal investment portfolio for an agent making decisions based on a utility function (see Merton (1969)). In this paper we deal with an investment problem for an investor who faces a stream of liabilities and makes decisions based on his/her exponential utility function. The exponential indifference price and hedging strategy for the payment process are next derived from the solution to the utility optimization problem.

We consider the Black-Scholes financial market with a bank account and a stock. We assume zero interest rate on the bank account, and random drift and volatility in the dynamics of the risky stock. This framework allows us to

2010 Mathematics Subject Classification: 91B16, 91B30, 93E20.

Key words and phrases: Black-Scholes model, random measure, backward stochastic differential equation, exponential utility, insurance and financial claims. 
model heavy-tailed asset returns and stochastic volatility. We investigate a stream of liabilities over a finite time horizon modelled by a payment process with claims occurring at random times, continuously over the duration of the contract and at the terminal time. The random times of the claims are generated by a random measure with a stochastic intensity of jumps. The claims are written on the asset traded in the financial market and on the non-tradeable source of risk driven by the random measure. The form of our payment process is motivated by Dahl et al. (2008) and Dahl and Møller (2006) who deal with a life insurance payment process under a stochastic mortality intensity. By allowing a stochastic jump intensity we can take into account both systematic and unsystematic claim intensity risk. We remark that by systematic claim intensity risk we mean unpredictable changes in the intensity of claims and by unsystematic claim intensity risk we understand randomness in the occurrence of claims. We recall that systematic risk is important in finance and insurance applications in which we should model a default intensity or a surrender intensity as a stochastic process related to the financial market.

In the financial mathematics literature the problems of exponential utility optimization and indifference pricing and hedging of contingent liabilities are well studied. For some recent results we refer to Becherer (2006), Bielecki et al. (2004), Frei and Schweizer (2009), Hu et al. (2005), Mania and Schweizer (2005) and references therein. These authors consider very general financial models with claims paid at the terminal time and derive theoretical existence results. Interestingly, in insurance mathematics an emphasis is put on indifference pricing and hedging of payment processes and claims paid over the duration of the contract. Less general but more explicit and applicable results are derived in the actuarial literature. We refer to Liang et al. (2011), Perera (2010), Wang (2007) and Wang et al. (2007) where the authors investigate the exponential utility optimization of the terminal wealth of an insurer who covers claims generated by a compound Poisson process over a finite time horizon. We also point out the work by Ludkovski and Young (2008) where the exponential indifference pricing and hedging problem is solved for a life insurer with a finite investment horizon who covers claims arising under life annuity and endowment contracts.

The goal of this paper is to solve the exponential utility optimization and indifference pricing and hedging problems for a general payment process and derive an explicit representation of the solution. We believe that there is a need to obtain pricing and hedging strategies for payment processes which are more sophisticated than a compound Poisson process. For example, the systematic claim intensity risk should be additionally included on the liability side. 
This paper completes and generalizes the results of Becherer (2006), Bielecki et al. (2004) and Delong (2010). In Becherer (2006) and Bielecki et al. (2004) the exponential utility optimization and indifference pricing and hedging problems for a terminal liability are solved, whereas in Delong (2010) the quadratic optimization and quadratic hedging problems for a payment process are solved. Here, we solve the exponential utility optimization and indifference pricing and hedging problems for the payment process. In order to solve our problems we apply backward stochastic differential equations (BSDEs) and we follow closely the works of Becherer $(2006)$ and $\mathrm{Hu}$ et al. (2005). We adapt the results from Becherer (2006) into our framework and make necessary reformulations and extensions in the proofs. As far as mathematics is concerned, we derive the BSDEs which characterize the optimal value function, the price and the investment strategy and we obtain the equivalent martingale measure which should be used in valuation of the claims in our general model. From the point of view of applications, our modifications allow us to state an explicit representation of the strategy which is of interest to an investor who aims at pricing and hedging of payment processes. We believe that our extension is important since in many applications, especially in insurance, investors deal with a stream of liabilities contingent on traded and non-traded risks which should be priced and hedged in a consistent way. Hence, the need for pricing and hedging results for general payment processes arises and we fill the gap in this respect. In particular, this is the first paper in the actuarial literature which provides an investment strategy for the insurer in the case when a policyholder lapses the contract under a stochastic intensity depending on the financial market.

This paper is structured as follows. In Section 2 we introduce the financial market and the payment process. The utility optimization problem is solved in Section 3. Section 4 deals with indifference pricing and hedging. The change of measure technique for a Brownian motion and a random measure is recalled in the Appendix.

2. The financial market and the payment process. Let us consider a probability space $(\Omega, \mathcal{F}, \mathbb{P})$ with a filtration $\mathcal{F}=\left(\mathcal{F}_{t}\right)_{0 \leq t \leq T}$ and a finite time horizon $T<\infty$. We assume that $\mathcal{F}$ satisfies the usual hypotheses of completeness $\left(\mathcal{F}_{0}\right.$ contains all sets of $\mathbb{P}$-measure zero) and right continuity $\left(\mathcal{F}_{t}=\mathcal{F}_{t+}\right)$.

We deal with the Black-Scholes financial model with stochastic coefficients. The financial market consists of two tradeable instruments: a bank account and a stock. The value of the bank account $S_{0}:=\left(S_{0}(t), 0 \leq t \leq T\right)$ is constant, $S_{0}(t)=1,0 \leq t \leq T$ (we assume zero interest rate) and the dynamics of the stock price $S:=(S(t), 0 \leq t \leq T)$ is given by the stochastic 
differential equation

$$
\frac{d S(t)}{S(t)}=\mu(t) d t+\sigma(t) d W(t), \quad S(0)=s_{0}>0,
$$

where $\mu:=(\mu(t), 0 \leq t \leq T)$ denotes the expected return on the stock, $\sigma:=$ $(\sigma(t), 0 \leq t \leq T)$ denotes the price volatility and $W:=(W(t), 0 \leq t \leq T)$ is an $\mathcal{F}$-adapted Brownian motion. We assume that

(A1) the processes $\mu, \sigma$ are $\mathcal{F}$-predictable and satisfy

$$
\begin{aligned}
& \int_{0}^{T}|\mu(t)| d t<\infty, \quad \int_{0}^{T}|\sigma(t)|^{2} d t<\infty, \quad \sigma(t)>0, \text { a.s. }, 0 \leq t \leq T, \\
& \left|\frac{\mu(t)}{\sigma(t)}\right| \leq K, \quad \text { a.s., } 0 \leq t \leq T .
\end{aligned}
$$

Moreover, the processes are predictable with respect to the natural filtration $\sigma(W(s), 0 \leq s \leq t)$.

If $\mu, \sigma$ are constant we obtain the classical Black-Scholes model. Our financial model with stochastic coefficients has a potential of taking into account economic factors, like stochastic volatility. The zero interest rate could be interpreted that we deal with discounted economic quantities.

We now introduce the payment process, following Delong (2010). Let $L:=(L(t), 0 \leq t \leq T)$ denote an $\mathcal{F}$-adapted step process. With $L$ we associate the random measure

$$
N(d t, d v)=\sum_{s \in(0, T]} \mathbf{1}_{(s, \triangle L(s))}(d t, d v) \mathbf{1}_{\{\triangle L(s) \neq 0\}}(s),
$$

with $\triangle L(s)=L(s)-L(s-)$, defined on $\Omega \times \mathcal{B}((0, T]) \times \mathcal{B}(\mathbb{R}-\{0\})$. For a Borel set $A$ the measure $N((0, t], A)$ counts the number of jumps of $L$ in the given set $A$ in the given period $(0, t]$. For details on random measures we refer to Chapter XI in He et al. (1992). We assume that

(A2) the random measure $N$ has a unique compensator $\vartheta$, defined on $\Omega \times \mathcal{B}((0, T]) \times \mathcal{B}(\mathbb{R}-\{0\})$, of the form

$$
\vartheta(d t, d v)=Q(t, d v) \eta(t) d t
$$

where $\eta: \Omega \times[0, T] \rightarrow[0, \infty)$ is a predictable process; for a fixed $(\omega, t) \in \Omega \times(0, T], Q(\omega, t, \cdot)$ is a probability measure on $\mathbb{R}-\{0\}$, and, for a fixed $A \in \mathcal{B}(\mathbb{R}-\{0\}), Q(\cdot, \cdot, A): \Omega \times[0, T] \rightarrow[0,1]$ is an $\mathcal{F}$-predictable process. The process $\eta$ satisfies

$$
\eta(t) \leq K, \quad \eta(t) \geq 0, \quad \text { a.s. }, 0 \leq t \leq T .
$$

The measure $Q$ satisfies

$$
Q(\omega, t, d v)=z(\omega, t, v) q(d v)
$$


with a density $z$ bounded a.s. a.e. in $(\omega, t, v)$, and a probability measure $q$.

We set $N(\{0\}, \mathbb{R}-\{0\})=N((0, T],\{0\})=\vartheta((0, T],\{0\})=0$. The assumption (A2) is useful in applications as $\eta$ stands for the intensity of jumps of the process $L$, and $Q$ gives the distribution of the jump's height given that the jump occurs. The boundedness of the density is the technical condition from Becherer (2006).

We deal with the stream of liabilities modelled by the payment process $P:=(P(t), 0 \leq t \leq T)$ described by

$$
P(t)=\int_{0}^{t} H(s) d s+\int_{0}^{t} \int_{\mathbb{R}} G(s, y) N(d s, d y)+F \mathbf{1}_{t=T} .
$$

The process $P$ contains payments $H$ which occur continuously during the term of the contract; it contains claims $G$ which occur at random times and the liability $F$ which is settled at the end of the contract (see Dahl and Møller (2006) and Dahl et al. (2008)). We assume that

(A3) the processes $H, G$ are $\mathcal{F}$-predictable and the random variable $F$ is $\mathcal{F}_{T}$-measurable. The processes $H, G$ and the random variable $F$ are non-negative and bounded a.s.

From the representation 2.1 and the fact that the step process $L$ has a finite number of jumps in $[0, T]$ we obtain

$$
\int_{0}^{t} \int_{\mathbb{R}} G(s, y) N(d s, d y)=\sum_{s \in(0, t]} G(s, \triangle L(s)) \mathbf{1}_{\{\triangle L(s) \neq 0\}}(s), \quad 0 \leq t \leq T,
$$

and the stochastic integral is well-defined. We conclude that the stochastic integral with respect to the random measure $N$ models claims occurring at the times when the step process $L$ jumps.

The payment process 2.2 is very general and may appear in various pricing and hedging problems in financial and insurance mathematics. Notice that the claims can depend (also in a pathwise sense) on different sources of uncertainty captured by the filtration $\mathcal{F}$. The liabilities $H, G, F$ can depend on the tradeable source of risk $W$ and on the non-tradeable source of risk $L$. We give four examples.

Example 1: A unit-linked life insurance. Choose

$$
\begin{aligned}
& \eta(t)=(n-L(t-)) \lambda(t), \quad Q(t,\{1\})=1, \\
& H(t)=h(t, S(t))(n-L(t-)), \quad G(t,\{1\})=g(t, S(t)), \\
& F=f(T, S(T))(n-L(T)) .
\end{aligned}
$$

The payment process $P$ models annuity, death and survival benefits from a portfolio consisting of $n$ insured persons whose lifetimes are independent and 
subject to a deterministic mortality intensity $\lambda$ (see Dahl and Møller (2006), Dahl et al. (2008) and Ludkovski and Young (2008)). We can investigate unit-linked products or variable annuities which are worldwide sold insurance products with financial guarantees.

EXAMPLE 2: An irrational lapse behaviour in life insurance. Under the choices (2.3) as in Example 1, let $\lambda$ be a stochastic intensity process adapted to the natural filtration $\sigma(W(s), 0 \leq s \leq t)$. The payment process $P$ models partial surrenders, total surrenders and survival benefits from a portfolio consisting of $n$ insured persons whose surrenders are conditionally independent and subject to the lapse intensity $\lambda$ related to the financial market. It is now known that a lapse behaviour of an insured depends on the financial market and such a dependence should be included in a pricing and hedging model (see European Commission QIS5 (2010)). In fact, an irrational lapse behaviour triggered by the performance of the financial market is one of the most important risks faced by life insurers.

ExAmple 3: A defaultable security. Under the same characteristics (2.3) the payment process $P$ could model claims from a portfolio of $n$ defaultable securities. In this framework defaults of securities are conditionally independent and subject to the default intensity $\lambda$ related to the financial market. Defaultable securities are extensively studied in the credit risk literature (see Bielecki et al. (2004) and Blanchet-Scalliet and Jeanblanc (2004)).

EXAmple 4: A collective insurance and credit risk model. Choose

$$
\begin{aligned}
& \eta(t)=\lambda, \quad Q(t, d v)=q(d v), \\
& H(t)=0, \quad G(t, v)=v, \quad F=0,
\end{aligned}
$$

where $q$ is a probability measure on a bounded support. The payment process $P$ is a compound Poisson process and it models claims occurring at a constant intensity $\lambda$ with a severity $q$. A compound Poisson process is the key process investigated in the collective insurance risk theory (see Liang et al. (2011), Perera (2010), Wang (2007), Wang et al. (2007)). More interestingly, we could take

$$
\begin{aligned}
& \eta(t)=\lambda(t), \quad Q(t, d v)=q(d v), \\
& H(t)=0, \quad G(t, v)=g(t, S(t), v), \quad F=0,
\end{aligned}
$$

and we could deal with the claims that are contingent on the stock $S$ and are generated by a Cox process with a stochastic claim intensity $\lambda$ adapted to the natural filtration $\sigma(W(s), 0 \leq s \leq t)$. Two important examples where we can use (2.4) concern weather (catastrophe) derivatives (see Ankirchner and Imkeller (2008)) and credit portfolio losses (see Gundlach and Lehrbass (2004)). 
We finally assume that under $(\mathbb{P}, \mathcal{F})$ the weak property of predictable representation holds (see Chapter XII.2 in He et al. (1992)), i.e.

(A4) every $(\mathbb{P}, \mathcal{F})$ local martingale $\mathcal{M}$ null at zero has a representation

$$
\mathcal{M}(t)=\int_{0}^{t} \psi(s) d W(s)+\int_{0}^{t} \int_{\mathbb{R}} \kappa(s, v) \tilde{N}(d s, d v), \quad 0 \leq t \leq T,
$$

with $\mathcal{F}$-predictable processes $(\psi, \kappa)$ integrable, in the sense of Itô calculus, with respect to the Brownian motion $W$ and the compensated random measure $\tilde{N}(d t, d v)=N(d t, d v)-\vartheta(d t, d v)$.

If $\mathcal{M}$ is square integrable then the representation (A4) is unique and the stochastic integrals are square integrable martingales. The assumption (A4) is usually satisfied in insurance and financial models (see Becherer $(2006)$ ). In particular, it is possible to construct the processes $(W, L)$ and take the filtration $\mathcal{F}$ as the natural filtration generated by these two driving processes to satisfy (A4) (see Becherer (2006) and He et al. (1992)).

3. Exponential utility optimization. First, we study exponential utility optimization of the terminal wealth.

The goal is to solve the optimization problem

$$
\sup _{\pi \in \mathcal{A}} \mathbb{E}\left[-e^{-\gamma X(T)}\right]
$$

for an investor who chooses an investment strategy $\pi$, rebalances his/her positions between the bank account $S_{0}$ and the stock $S$ and covers the stream of liabilities $P$. The investor's wealth process $X:=(X(t), 0 \leq t \leq T)$ is given by the stochastic differential equation

$$
\begin{aligned}
d X(t)= & \pi(t)(\mu(t) d t+\sigma(t) d W(t))-H(t) d t-\int_{\mathbb{R}} G(t, v) N(d t, d v) \\
& -F d \mathbf{1}\{t \geq T\}, \quad X(0)=x,
\end{aligned}
$$

where $x$ denotes an initial capital. The investment strategy $\pi$ can be chosen from a class $\mathcal{A}$ of admissible strategies.

Definition 3.1. The class $\mathcal{A}$ of admissible investment strategies consists of $\mathcal{F}$-predictable processes $\pi:=(\pi(t), 0 \leq t \leq T)$ such that

$$
\begin{aligned}
& \mathbb{E}\left[\int_{0}^{T}|\pi(s) \sigma(s)|^{2} d s\right]<\infty, \\
& \left\{e^{-\gamma X^{\pi}(\tau)}: \tau \text { an } \mathcal{F} \text {-stopping time }\right\} \text { is } \mathbb{P} \text {-uniformly integrable. }
\end{aligned}
$$

The class of strategies from Definition 1.1 usually arises when dealing with exponential utility optimization and it excludes arbitrage strategies (see Becherer (2006) and Hu et al. (2005) for details). 
Consider the backward stochastic differential equation

$$
\begin{aligned}
Y(t)= & F+\int_{t}^{T} f(s) d s-\int_{t}^{T} Z(s) d W(s) \\
& -\int_{t}^{T} \int_{\mathbb{R}} U(s, v) \tilde{N}(d s, d v), \quad 0 \leq t \leq T,
\end{aligned}
$$

where the generator $f$ will be specified later. We use the BSDE (3.3) to solve our optimization problem (3.1).

Consider the process $V:=(V(t), 0 \leq t \leq T)$ with the dynamics

$$
\begin{aligned}
d V(t)= & \pi(t)(\mu(t) d t+\sigma(t) d W(t))-H(t) d t-\int_{\mathbb{R}} G(t, v) \tilde{N}(d t, d v) \\
& -\int_{\mathbb{R}} G(t, v) Q(t, d v) \eta(t) d t, \quad V(0)=x
\end{aligned}
$$

and notice that our optimization criterion is equivalent to

$$
\mathbb{E}\left[-e^{-\gamma X(T)}\right]=\mathbb{E}\left[-e^{-\gamma(V(T)-Y(T))}\right] .
$$

The idea of solving 3.1), proposed in $\mathrm{Hu}$ et al. (2005) and extended in Becherer (2006), is to find a generator $f$ of the BSDE (3.3), independent of $\pi$, such that the process $A:=(A(t), 0 \leq t \leq T)$ defined by

$$
A^{\pi}(t)=-e^{-\gamma\left(V^{\pi}(t)-Y(t)\right)}, \quad 0 \leq t \leq T,
$$

is a martingale for some $\pi^{*} \in \mathcal{A}$ and a supermartingale for all $\pi \in \mathcal{A}$. If $A$ is a supermartingale for any $\pi \in \mathcal{A}$, we obtain

$$
\mathbb{E}\left[A^{\pi}(T)\right]=\mathbb{E}\left[-e^{-\gamma\left(V^{\pi}(T)-Y(T)\right)}\right] \leq A(0),
$$

and if $A$ is a martingale for $\pi^{*} \in \mathcal{A}$, we conclude that

$\mathbb{E}\left[A^{\pi}(T)\right]=\mathbb{E}\left[-e^{-\gamma\left(V^{\pi}(T)-Y(T)\right)}\right] \leq A(0)=\mathbb{E}\left[-e^{-\gamma\left(V^{\pi^{*}}(T)-Y(T)\right)}\right]=\mathbb{E}\left[A^{\pi^{*}}(T)\right]$, and the optimality of $\pi^{*}$ follows.

We can find $\left(f, \pi^{*}\right)$ in the following way. By (3.3) and (3.4) we obtain

$$
\begin{gathered}
-\gamma(V(t)-Y(t))=-\gamma\left(X(0)+\int_{0}^{t} \pi(s) \mu(s) d s+\int_{0}^{t} \pi(s) \sigma(s) d W(s)\right. \\
-\int_{0}^{t} H(s) d s-\int_{0}^{t} \int_{\mathbb{R}} G(s, v) Q(s, d v) \eta(s) d s-\int_{0}^{t} \int_{\mathbb{R}} G(s, v) \tilde{N}(d s, d v) \\
\left.-Y(0)+\int_{0}^{t} f(s) d s-\int_{0}^{t} Z(s) d W(s)-\int_{0}^{t} \int_{\mathbb{R}} U(s, v) \tilde{N}(d s, d v)\right)
\end{gathered}
$$




$$
\begin{aligned}
& =-\gamma(X(0)-Y(0))-\gamma \int_{0}^{t} \pi(s) \mu(s) d s-\gamma \int_{0}^{t}(\pi(s) \sigma(s)-Z(s)) d W(s) \\
& +\gamma \int_{0}^{t}\left(H(s)+\int_{\mathbb{R}} G(s, v) Q(s, d v) \eta(s)\right) d s \\
& +\gamma \int_{0}^{t}(G(s, v)+U(s, v)) \tilde{N}(d s, d v)-\gamma \int_{0}^{t} f(s) d s, \quad 0 \leq t \leq T .
\end{aligned}
$$

We define the processes $M^{W}:=\left(M^{W}(t), 0 \leq t \leq T\right)$ and $M^{N}=\left(M^{N}(t)\right.$, $0 \leq t \leq T)$ by

$$
\begin{aligned}
M^{W}(t)= & e^{-\gamma \int_{0}^{t}(\pi(s) \sigma(s)-Z(s)) d W(s)-\frac{1}{2} \gamma^{2} \int_{0}^{t}(\pi(s) \sigma(s)-Z(s))^{2} d s}, \\
M^{N}(t)= & e^{\gamma \int_{0}^{t} \int_{\mathbb{R}}(G(s, v)+U(s, v)) \tilde{N}(d s, d v)} \\
& \cdot e^{\left.+\int_{0}^{t} \int_{\mathbb{R}}\left(\gamma(G(s, v)+U(s, v))-e^{\gamma(G(s, v)+U(s, v))}+1\right) Q(s, d v) \eta(s) d s\right)},
\end{aligned}
$$

the process $D:=(D(s), 0 \leq s \leq T)$ by

$$
\begin{aligned}
D(s) & =-\gamma \pi(s) \mu(s)+\frac{1}{2} \gamma^{2}(\pi(s) \sigma(s)-Z(s))^{2} \\
& +\gamma\left(H(s)+\int_{\mathbb{R}} G(s, v) Q(s, d v) \eta(s)\right) \\
& -\int_{\mathbb{R}}\left(\gamma(G(s, v)+U(s, v))-e^{\gamma(G(s, v)+U(s, v))}+1\right) Q(s, d v) \eta(s)-\gamma f(s),
\end{aligned}
$$

and we obtain the relation

$$
\begin{aligned}
A(t) & =-e^{-\gamma(V(t)-Y(t))} \\
& =-M^{W}(t) M^{N}(t) e^{-\gamma(X(0)-Y(0))+\int_{0}^{t} D(s) d s}, \quad 0 \leq t \leq T .
\end{aligned}
$$

First, we choose a strategy $\pi^{*}$ to minimize

$$
\min _{\pi}\left\{-\gamma \pi(s) \mu(s)+\frac{1}{2} \gamma^{2}(\pi(s) \sigma(s)-Z(s))^{2}\right\} .
$$

and we get

$$
\pi^{*}(s)=\frac{1}{\sigma(s)}\left(Z(s)+\frac{\mu(s)}{\gamma \sigma(s)}\right), \quad 0 \leq s \leq T .
$$

Second, we choose the generator $f$,

$$
\begin{aligned}
f(s)= & -\pi^{*}(s) \mu(s) d s+\frac{1}{2} \gamma\left(\pi^{*}(s) \sigma(s)-Z(s)\right)^{2} d s \\
& +\left(H(s)+\int_{\mathbb{R}} G(s, v) Q(s, d v) \eta(s)\right) d s
\end{aligned}
$$




$$
\begin{aligned}
& -\frac{1}{\gamma} \int_{\mathbb{R}}\left(\gamma(G(s, v)+U(s, v))-e^{\gamma(G(s, v)+U(s, v))}+1\right) Q(s, d v) \eta(s) \\
= & -\frac{\mu^{2}(s)}{2 \gamma \sigma^{2}(s)}-\frac{\mu(s)}{\sigma(s)} Z(s)+H(s) \\
& +\int_{\mathbb{R}}\left(\frac{1}{\gamma}\left(e^{\gamma(G(s, v)+U(s, v))}-1\right)-U(s, v)\right) Q(s, d v) \eta(s), \quad 0 \leq s \leq T .
\end{aligned}
$$

Notice that $\left(f, \pi^{*}\right)$ have been chosen such that for any $\pi$ we have $D(s) \geq 0$, $0 \leq s \leq T$, and for $\pi^{*}$ we have $D(s)=0,0 \leq s \leq T$. Moreover, $f$ is independent of $\pi$. The above heuristic reasoning has now to be made more formal.

Theorem 3.1. Assume that (A1)-(A4) hold. The strategy

$$
\pi^{*}(t)=\frac{1}{\sigma(t)}\left(Z(t)+\frac{\mu(t)}{\gamma \sigma(t)}\right), \quad 0 \leq t \leq T,
$$

where $(Y, Z, U)$ solve the backward stochastic differential equation

$$
\begin{aligned}
Y(t)= & F+\int_{t}^{T}\left(-\frac{\mu^{2}(s)}{2 \gamma \sigma^{2}(s)}-\frac{\mu(s)}{\sigma(s)} Z(s)+H(s)\right. \\
& \left.+\int_{\mathbb{R}}\left(\frac{1}{\gamma}\left(e^{\gamma(G(s, v)+U(s, v))}-1\right)-U(s, v)\right) Q(s, d v) \eta(s)\right) d s \\
& -\int_{t}^{T} Z(s) d W(s)-\int_{t}^{T} U(s, v) \tilde{N}(d s, d v), \quad 0 \leq t \leq T,
\end{aligned}
$$

is an admissible optimal investment strategy for the utility optimization problem (3.1). The value function of the optimization problem (3.1) at time $t=0$ equals $-e^{-\gamma(x-Y(0))}$.

Proof. We divide the proof into several steps.

1. The existence and uniqueness of a solution to the BSDE (3.9). We introduce the measure

$$
\frac{d \mathbb{Q}^{F}}{d \mathbb{P}} \mid \mathcal{F}_{t}=e^{-\int_{0}^{t} \frac{\mu(s)}{\sigma(s)} d W(s)-\frac{1}{2} \int_{0}^{t}\left|\frac{\mu(s)}{\sigma(s)}\right|^{2} d s}, \quad 0 \leq t \leq T .
$$

The measure $\mathbb{Q}^{F}$ is a probability measure as $\mu(s) / \sigma(s)$ is uniformly bounded in $s$, by the assumption (A1). We change the measure in the BSDE (3.9) and we arrive at 


$$
\begin{aligned}
Y(t)= & F+\int_{t}^{T}\left(-\frac{\mu^{2}(s)}{2 \gamma \sigma^{2}(s)}+H(s)\right. \\
& \left.+\int_{\mathbb{R}}\left(\frac{1}{\gamma}\left(e^{\gamma(G(s, v)+U(s, v))}-1\right)-U(s, v)\right) Q(s, d v) \eta(s)\right) d s \\
& -\int_{t}^{T} Z(s) d W^{\mathbb{Q}^{F}}(s)-\int_{t}^{T} U(s, v) \tilde{N}^{\mathbb{Q}^{F}}(d s, d v), \quad 0 \leq t \leq T .
\end{aligned}
$$

We can divide the generator $f$ in 3.10 into two parts:

$$
\begin{aligned}
f(s) & =\hat{f}(s)+\int_{\mathbb{R}} \bar{f}(U(s, v)) Q(s, d v) \eta(s) \\
\hat{f}(s) & =-\frac{\mu^{2}(s)}{\gamma \sigma^{2}(s)}+H(s)+\frac{1}{\gamma} \int_{\mathbb{R}}\left(e^{\gamma G(s, v)}-1\right) Q(s, d v) \eta(s), \\
\bar{f}(U(s, v)) & =\frac{1}{\gamma}\left(e^{\gamma(G(s, v)+U(s, v))}-e^{\gamma G(s, v)}\right)-U(s, v) .
\end{aligned}
$$

By Theorem 3.5 in Becherer $(2006)$, we now conclude that the BSDE (3.10) has a unique solution under $\mathbb{Q}^{F^{\prime}}$ with $\mathcal{F}$-adapted $Y$ and $\mathcal{F}$-predictable $(Z, U)$ such that

$$
\begin{aligned}
& \mathbb{E}^{\mathbb{Q}^{F}}\left[\int_{0}^{T}|Z(s)|^{2} d s\right]<\infty, \\
& |Y(s)|+|U(s, v)| \leq K, \mathbb{Q}^{F} \text {-a.s., a.e. } s \in[0, T], v \in \mathbb{R} .
\end{aligned}
$$

Moreover, by Lemma 3.4 in Becherer $(2006)$, the process $\int_{0}^{t} Z(s) d W^{\mathbb{Q}^{F}}(s)$ is a $\operatorname{BMO}\left(\mathbb{Q}^{F}\right)$-martingale.

Consider the following change of measure:

$$
\frac{d \mathbb{P}}{d \mathbb{Q}^{F}} \mid \mathcal{F}_{t}=e^{\int_{0}^{t} \frac{\mu(s)}{\sigma(s)} d W^{\mathbb{Q}^{F}}(s)-\frac{1}{2} \int_{0}^{t}\left|\frac{\mu(s)}{\sigma(s)}\right|^{2} d s}, \quad 0 \leq t \leq T,
$$

which is defined as the stochastic exponential of the martingale $M^{F F}(t)=$ $\int_{0}^{t} \frac{\mu(s)}{\sigma(s)} d W^{\mathbb{Q}^{F}}(s)$. The martingale $M^{F F}$ is a $\operatorname{BMO}\left(\mathbb{Q}^{F}\right)$-martingale, as

$$
\sup _{\tau \text { an } \mathcal{F} \text {-stopping time }} \mathbb{E}^{\mathbb{Q}^{F}}\left[\int_{\tau}^{T}\left|\frac{\mu(s)}{\sigma(s)}\right|^{2} d s \mid \mathcal{F}_{\tau}\right]<\infty
$$

by the boundedness assumption (A1). Theorem 3.6 in Kazamaki (1994) now implies that $\int_{0}^{t} Z(s) d W(s)$ is a $\mathrm{BMO}(\mathbb{P})$-martingale (see also Becherer (2006) and $\mathrm{Hu}$ et al. (2005)). We can finally conclude that the BSDE (3.10) has a unique solution under $\mathbb{P}$ with $\mathcal{F}$-adapted $Y$ and $\mathcal{F}$-predictable $(Z, U)$ such 
that

$$
\begin{aligned}
& \mathbb{E}^{\mathbb{P}}\left[\int_{0}^{T}|Z(s)|^{2} d s\right]<\infty, \\
& |Y(s)|+|U(s, v)| \leq K, \quad \mathbb{P} \text {-a.s., a.e. } s \in[0, T], v \in \mathbb{R} .
\end{aligned}
$$

Clearly, the solution $(Y, Z, U)$ does not depend on $\pi$.

2. The supermartingale property. By (3.2), 3.11) and the boundedness assumptions (A2)-(A3), the processes $M^{W}, M^{N}$ defined in (3.5) are stochastic exponentials of local martingales and they have the following dynamics

$$
\begin{aligned}
& \frac{d M^{W}(t)}{M^{W}(t)}=-\gamma(\pi(t) \sigma(t)-Z(t)) d W(t), \\
& \frac{d M^{N}}{M^{N}(t-)}=\int_{\mathbb{R}}\left(e^{\gamma(G(t, v)+U(t, v))}-1\right) \tilde{N}(d t, d v) .
\end{aligned}
$$

Consider the process $M:=(M(t), 0 \leq t \leq T)$ where

$$
M(t)=M^{W}(t) M^{N}(t), \quad 0 \leq t \leq T,
$$

and by Itô's formula we obtain its dynamics

$$
\begin{aligned}
\frac{d M(t)}{M(t)}= & -\gamma(\pi(t) \sigma(t)-Z(t)) d W(t) \\
& +\int_{\mathbb{R}}\left(e^{\gamma(G(t, v)+U(t, v))}-1\right) \tilde{N}(d t, d v) .
\end{aligned}
$$

For any $\pi \in \mathcal{A}$ the process $M$ is a local martingale. Moreover, as $e^{\gamma(G(t, v)+U(t, v))}$ $-1>-1$ we conclude that the martingale $M$ is positive. Our assumptions also imply that for any $\pi \in \mathcal{A}$ the process $D$ is integrable and $\int_{0}^{T} D(s) d s<\infty$ a.s.

Recall now the relation 3.6,

$$
A(t)=-e^{-\gamma(V(t)-Y(t))}=-M(t) e^{-\gamma(X(0)-Y(0))+\int_{0}^{t} D(s) d s}, \quad 0 \leq t \leq T,
$$

which is well-defined a.s. As $M$ is a positive local martingale and $D(s) \geq 0$, we can derive

$$
\begin{aligned}
& \mathbb{E}\left[A\left(t \wedge \tau_{n}\right) \mid \mathcal{F}_{s}\right]=\mathbb{E}\left[-M\left(t \wedge \tau_{n}\right) e^{-\gamma(X(0)-Y(0))+\int_{0}^{t \wedge \tau_{n}} D(u) d u} \mid \mathcal{F}_{s}\right] \\
\leq & \mathbb{E}\left[-M\left(t \wedge \tau_{n}\right) \mid \mathcal{F}_{s}\right] e^{-\gamma(X(0)-Y(0))+\int_{0}^{s \wedge \tau_{n}} D(u) d u} \\
= & -M\left(s \wedge \tau_{n}\right) e^{-\gamma(X(0)-Y(0))+\int_{0}^{s \wedge \tau_{n}} D(u) d u}=A\left(s \wedge \tau_{n}\right), \quad 0 \leq s \leq t \leq T,
\end{aligned}
$$

where $\left(\tau_{n}\right)_{n \in \mathbb{N}}$ denotes a localizing sequence for the local martingale $M$. Notice that

$$
A^{\pi}(t)=-e^{-\gamma\left(V^{\pi}(t)-Y(t)\right)}=-e^{-\gamma\left(X^{\pi}(t)+\mathbf{1}\{t=T\} F-Y(t)\right)}, \quad 0 \leq t \leq T,
$$


and by uniform integrability of the family $e^{-\gamma X^{\pi}(\tau)}$ assumed in 3.2 , together with boundedness of $F$ and of the solution $Y$, we conclude that the family $A^{\pi}(\tau)$ for $\mathcal{F}$-stopping times $\tau$ is uniformly integrable. Letting $n \rightarrow \infty$ in 3.13 we obtain the supermartingale property of $A$ for any $\pi \in \mathcal{A}$.

3. The martingale property. We now prove that $A^{\pi}$ is a martingale for $\pi^{*}$ defined in (3.8). First, we have $D(s)=0$ for $0 \leq s \leq T$. Next, we investigate the local martingale $M$ from (3.12). Define $\tau_{n}=\inf \{t:|M(t)|>n\}$. The process $M$ under $\pi^{*}$ takes the form

$$
\begin{aligned}
M(t)= & 1-\int_{0}^{t} \gamma M(s-) \frac{\mu(s)}{\gamma \sigma(s)} d W(s) \\
& +\gamma \int_{0}^{t} \int_{\mathbb{R}} M(s-)\left(e^{\gamma(G(s, v)+U(s, v))}-1\right) \tilde{N}(d s, d v), \quad 0 \leq t \leq T .
\end{aligned}
$$

By the Cauchy-Schwarz inequality, the property that our localized stochastic integrals are square integrable martingales, the boundedness assumptions and the properties of the solution to the BSDE we obtain

$$
\begin{aligned}
\mathbb{E}\left[|M(t)|^{2} \mathbf{1}\left\{t \leq \tau_{n}\right\}\right] & \leq \mathbb{E}\left[\left|M\left(t \wedge \tau_{n}\right)\right|^{2}\right] \\
& \leq K\left(1+\int_{0}^{t} \mathbb{E}\left[|M(s)|^{2} \mathbf{1}\left\{s \leq \tau_{n}\right\}\right] d s\right), \quad 0 \leq t \leq T
\end{aligned}
$$

with $K$ independent of $n$ and $t$. By Gronwall's inequality we finally arrive at

$$
\mathbb{E}\left[|M(t)|^{2} \mathbf{1}\left\{t \leq \tau_{n}\right\}\right] \leq K e^{K T} .
$$

Letting $n \rightarrow \infty$, together with Fatou's lemma, gives $\sup _{t \in[0, T]} \mathbb{E}\left[|M(t)|^{2}\right]$ $<\infty$. We conclude that the local martingale $M$ is a square integrable martingale. By (3.6) this proves that $A^{\pi}$ is a martingale for $\pi^{*}$. Items 2-3 yield optimality of the candidate strategy $\pi^{*}$.

4. Admissibility of the candidate strategy. The strategy $\pi^{*}$ is $\mathcal{F}$-predictable as $Z, \mu, \sigma$ are $\mathcal{F}$-predictable, and it is square integrable $(3.2)$ as $Z$ is square integrable and $\mu(t) / \sigma(t)$ is uniformly bounded in $t$. The family $\left\{e^{-\gamma X^{\pi^{*}}(\tau)}\right.$ : $\tau$ an $\mathcal{F}$-stopping time $\}$ is uniformly integrable as

$$
e^{-\gamma X^{*}(\tau)}=M(\tau) e^{\gamma(\mathbf{1}\{\tau=T\} F-Y(\tau))-\gamma(X(0)-Y(0))}, \quad \text { a.s. },
$$

and $M$ is uniformly integrable and $F, Y$ are bounded.

4. Exponential indifference pricing and hedging. We now investigate the exponential indifference pricing and hedging problem for the stream of liabilities $P$. The solution is based on Theorem 3.1. 
The indifference price process $\mathcal{Y}:=(\mathcal{Y}(t), 0 \leq t \leq T)$, related to the payment process $P$, is defined as a solution to the equation

$$
\mathcal{V}^{P}(t, x+\mathcal{Y}(t))=\mathcal{V}^{0}(t, x), \quad 0 \leq t \leq T,
$$

where $\mathcal{V}(t, x)$ denotes the optimal value function of the dynamic utility optimization problem (3.1) at time $t$ given that $X(t)=x$. The price $\mathcal{Y}$ from (4.1) makes the investor indifferent at time $t$ between issuing or taking over the contract maturing at $T>t$, collecting the premium $\mathcal{Y}(t)$, covering the future claims arising from $P$ and not issuing or taking over the contract. We are interested in deriving the indifference price process $\mathcal{Y}$ under the exponential utility function. The indifference hedging strategy could next be deduced from the form of $\mathcal{Y}$.

Theorem 4.1. Assume that (A1)-(A4) hold. The exponential indifference price process $\mathcal{Y}:=(\mathcal{Y}(t), 0 \leq t \leq T)$ solves the backward stochastic differential equation

$$
\begin{aligned}
\mathcal{Y}(t)= & F+\int_{t}^{T}\left(-\frac{\mu(s)}{\sigma(s)} \mathcal{Z}(s)+H(s)\right. \\
& \left.+\int_{\mathbb{R}}\left(\frac{1}{\gamma}\left(e^{\gamma(G(s, v)+\mathcal{U}(s, v))}-1\right)-\mathcal{U}(s, v)\right) Q(s, d v) \eta(s)\right) d s \\
& -\int_{t}^{T} \mathcal{Z}(s) d W(s)-\int_{t}^{T} \int_{\mathbb{R}} \mathcal{U}(s, v) \tilde{N}(d s, d v), \quad 0 \leq t \leq T .
\end{aligned}
$$

The exponential indifference price can be represented as

$$
\mathcal{Y}(t)=\mathbb{E}^{\mathbb{Q}^{P}}\left[\int_{t}^{T} d P(t) \mid \mathcal{F}_{t}\right], \quad 0 \leq t \leq T,
$$

where the expected value is taken under an equivalent martingale measure $\mathbb{Q}^{P}$ arising from the following change of measure:

$$
\begin{aligned}
& \frac{d \mathbb{Q}^{P}}{d \mathbb{P}} \mid \mathcal{F}_{T}=M(T), \\
& \frac{d M^{P}(t)}{M^{P}(t-)}=-\frac{\mu(t)}{\sigma(t)} d W(t)+\int_{\mathbb{R}}\left(\frac{e^{\gamma(G(t, v)+\mathcal{U}(t, v))}-1}{\gamma(G(t, v)+\mathcal{U}(t, v))}-1\right) \tilde{N}(d t, d v) .
\end{aligned}
$$

Proof. Again, the proof is in several steps.

1. The indifference price as a solution to the BSDE 4.2. Based on Theorem 3.1 we find that the process $\mathcal{Y}$ satisfies

$$
-e^{-\gamma\left(x+\mathcal{Y}(t)-Y^{P}(t)\right)}=-e^{-\gamma\left(x-Y^{0}(t)\right)}, \quad 0 \leq t \leq T .
$$

We arrive at

$$
\mathcal{Y}(t)=Y^{P}(t)-Y^{0}(t), \quad 0 \leq t \leq T,
$$


where $Y^{P}$ denotes the solution to the BSDE (3.3) with the generator (3.7), and $Y^{0}$ is a solution to the BSDE

$$
\begin{aligned}
Y^{0}(t)= & \int_{t}^{T}\left(\frac{-\mu^{2}(s)}{2 \gamma \sigma^{2}(s)}-\frac{\mu(s)}{\sigma(s)} Z^{0}(s)\right) d s \\
& -\int_{t}^{T} Z^{0}(s) d W(s)-\int_{t}^{T} U^{0}(s, v) \tilde{N}(d s, d v), \quad 0 \leq t \leq T,
\end{aligned}
$$

which corresponds to the utility optimization problem 3.1 without the payment process $P$ (a pure investment problem). The BSDE (4.4) is a linear equation and Proposition 3.2 from Becherer (2006) shows immediately that there exists a unique solution to 4 .4 with $\mathcal{F}$-adapted $Y^{0}$ and $\mathcal{F}$-predictable $Z^{0}$ such that

$$
\begin{aligned}
& \mathbb{E}\left[\int_{0}^{T}\left|Z^{0}(s)\right|^{2} d s\right]<\infty, \\
& \left|Y^{0}(t)\right|+\left|U^{0}(s, v)\right| \leq K, \quad \text { a.s., a.e. } s \in[0, T], v \in \mathbb{R} .
\end{aligned}
$$

Moreover, by the measurability assumption (A1) we can set $U^{0}(s, v)=0$. By substituting $Y^{P}$ and $Y^{0}$ we can derive that the indifference price process $\mathcal{Y}$ satisfies the BSDE (4.2), where we introduce the processes $\mathcal{Z}=Z^{P}-Z^{0}$ and $\mathcal{U}=U^{P}-U^{0}$.

2. The representation of the price under an equivalent martingale measure. Notice that the processes

$$
\begin{aligned}
& \int_{0}^{t} \frac{\mu(s)}{\sigma(s)} d W(s), \quad 0 \leq t \leq T, \\
& \int_{0}^{t} \int_{\mathbb{R}}\left(\frac{e^{\gamma(G(s, v)+\mathcal{U}(s, v))}-1}{\gamma(G(s, v)+\mathcal{U}(s, v))}-1\right) \tilde{N}(d s, d v), \quad 0 \leq t \leq T,
\end{aligned}
$$

are square integrable martingales as the integrands are a.s. bounded. We remark that the function $r(y)=\left(e^{y}-1\right) / y-1$ is continuous, bounded, $|r(y)| \leq e^{|y|}+1$, with $r(y)>-1$ and $r(0)=0$. Hence, we can define a local martingale $M^{P}:=\left(M^{P}(t), 0 \leq t \leq T\right)$ as the stochastic exponential

$$
\frac{d M^{P}(t)}{M^{P}(t-)}=-\frac{\mu(t)}{\sigma(t)} d W(t)+\int_{\mathbb{R}}\left(\frac{e^{\gamma(G(t, v)+\mathcal{U}(t, v))}-1}{\gamma(G(t, v)+\mathcal{U}(t, v))}-1\right) \tilde{N}(d t, d v)
$$

As in 3.14 , we can show that $M^{P}$ defined in 4.6 is a square integrable martingale. The process $M^{P}$ is positive. We conclude that $M^{P}$ defines an equivalent probability measure $\mathbb{Q}^{P}$. Moreover, $\mathbb{Q}^{P}$ is an equivalent martingale measure as the process $S$ is a $\mathbb{Q}^{P}$-martingale. 
By changing the measure in 4.2 we find that the indifference price process $\mathcal{Y}$ satisfies the BSDE

$$
\begin{aligned}
\mathcal{Y}(t)= & F+\int_{t}^{T}\left(H(s)+\int_{\mathbb{R}} G(s, v)(1+\kappa(s, v)) Q(s, d v) \eta(s)\right) d s \\
& -\int_{t}^{T} \mathcal{Z}(s) d W^{\mathbb{Q}^{P}}(s)-\int_{t}^{T} \int_{\mathbb{R}} \mathcal{U}(s, v) \tilde{N}^{\mathbb{Q}^{P}}(d s, d v), \quad 0 \leq t \leq T .
\end{aligned}
$$

The BSDE (4.7) has a simple structure and we can establish a probabilistic representation of $\mathcal{Y}$. Notice that by Burkholder's inequality we have

$$
\mathbb{E}^{\mathbb{Q}^{P}}\left[\sup _{t \in[0, T]}\left|\int_{0}^{t} \mathcal{Z}(s) d W^{\mathbb{Q}^{P}}(s)\right|\right] \leq K \mathbb{E}^{\mathbb{Q}^{P}}\left[\left(\int_{0}^{T}|\mathcal{Z}(s)|^{2} d s\right)^{1 / 2}\right],
$$

and by the Cauchy-Schwarz inequality we obtain

$$
\mathbb{E}^{\mathbb{Q}^{P}}\left[\left(\int_{0}^{T}|\mathcal{Z}(s)|^{2} d s\right)^{1 / 2}\right] \leq K\left(\mathbb{E}\left[\left|M^{P}(T)\right|^{2}\right] \mathbb{E}\left[\int_{0}^{T}|\mathcal{Z}(s)|^{2} d s\right]\right)^{1 / 2}<\infty,
$$

where we use square integrability of $M, Z^{P}, Z^{0}$ under $\mathbb{P}$. The inequalities 4.8 and 4.9) imply that the $\mathbb{Q}^{P}$-local martingale $\int_{0}^{t} \mathcal{Z}(s) d W^{\mathbb{Q}^{P}}(s)$ is a $\mathbb{Q}^{P}$-martingale. In the same way we can prove that the $\mathbb{Q}^{P}$-local martingale $\int_{0}^{t} \int_{\mathbb{R}} \mathcal{U}(s, v) \tilde{N}^{\mathbb{Q}^{P}}(d s, d v)$ is a $\mathbb{Q}^{P}$-martingale. Taking the expected value in (4.7) we obtain the key representation

$$
\begin{aligned}
\mathcal{Y}(t) & =\mathbb{E}^{\mathbb{Q}^{P}}\left[F+\int_{t}^{T}\left(H(s)+\int_{\mathbb{R}} G(s, v)(1+\kappa(s, v)) Q(s, d v) \eta(s)\right) d s \mid \mathcal{F}_{t}\right] \\
& =\mathbb{E}^{\mathbb{Q}^{P}}\left[F+\int_{t}^{T}\left(H(s)+\int_{\mathbb{R}} G(s, v) N(d s, d v)\right) d s \mid \mathcal{F}_{t}\right], \\
& =\mathbb{E}^{\mathbb{Q}^{P}}\left[\int_{t}^{T} d P(t) \mid \mathcal{F}_{t}\right], \quad 0 \leq t \leq T,
\end{aligned}
$$

where we use the compensating property (5.1) for the random measure $N$ under $\mathbb{Q}^{P}$.

The representation 4.3 shows that the indifference price $\mathcal{Y}$ for the payment process $P$ does not induce an arbitrage. The indifference price is represented as the expected value of the cash flows arising from the stream of liabilities $P$ under an equivalent martingale measure. Recall that in an incomplete market there exist many prices and equivalent martingale measures. The exponential pricing principle determines the equivalent martingale measure which should be used for pricing. Notice that the martingale measure $\mathbb{Q}^{P}$ depends on the financial market and on the liability $P$ which is not traded in the market. Theorems 3.1 and 4.1 extend the results from Becherer (2006) and Bielecki et al. (2004). 
Proposition 4.1. Under the assumptions of Theorem 4.1, the indifference hedging strategy takes the form

$$
\Pi(t)=\frac{\mathcal{Z}(t)}{\sigma(t)}, \quad 0 \leq t \leq T .
$$

Following Ankirchner et al. (2010) and Becherer (2006) we can call the process

$$
\Pi(t)=\frac{\mathcal{Z}(t)}{\sigma(t)}=\frac{Z^{P}(t)-Z^{0}(t)}{\sigma(t)}
$$

the indifference hedging strategy for the stream of liabilities $P$. The process $\Pi$ represents the change in the optimal investment strategy which makes the investor indifferent with respect to $P$. From the representation (4.7) we could interpret $\Pi$ as the amount which should be dynamically invested in $S$ to hedge partially the cash flows from $P$. Notice that we can arrive at

$$
\begin{aligned}
\mathcal{Y}(t)= & \mathcal{Y}(0)+\int_{0}^{t} \Pi(s)(\mu(s)+\sigma(s) d W(s))-P(t) \\
& +\int_{0}^{t} \int_{\mathbb{R}}(\mathcal{U}(s, v)+G(s, v)) \tilde{N}^{\mathbb{Q}^{P}}(d s, d v), \quad 0 \leq t \leq T,
\end{aligned}
$$

which confirms the last remark. The integral with respect to the random measure could be interpreted as additional cash inflows/outflows, over the gains from the self-financing hedging strategy $\Pi$, which are needed to cover the past liabilities and to bring the wealth process to the required (statutory) level of the expected value of the future liabilities.

5. Conclusion. We have solved the exponential utility optimization and indifference pricing and hedging problems for a general payment process. We believe that the results obtained could be useful for investors in setting prices of liabilities and hedging strategies in their advanced risk management models which are advocated in the Basel II and Solvency II Directives.

A. Appendix. We recall the measure change technique for a Brownian motion and a random measure. Take $\mathcal{F}$-predictable processes $\psi:=(\psi(t)$, $0 \leq t \leq T), \kappa:=(\kappa(t, v), 0 \leq t \leq T, v \in \mathbb{R})$ such that

$$
\begin{aligned}
& \int_{0}^{T}|\psi(t)|^{2} d t<\infty, \\
& \int_{0}^{T} \int_{\mathbb{R}}|\kappa(t, v)|^{2} Q(t, d v) \eta(t) d t<\infty, \quad \kappa(t, v)>-1, \quad 0 \leq t \leq T, v \in \mathbb{R} .
\end{aligned}
$$

Consider the process $M:=(M(t), 0 \leq t \leq T)$ with the dynamics 


$$
\frac{d M(t)}{M(t-)}=\psi(t) d W(t)+\int_{\mathbb{R}} \kappa(t, v) \tilde{N}(d t, d v), \quad M(0)=1 .
$$

This process is a positive local martingale (see Chapter II.8 in Protter $(2004))$. Choose $(\psi, \kappa)$ such that $\mathbb{E}[M(t)]=1$ for all $t \in[0, T]$, so that $M$ is a true martingale. With this choice of $(\psi, \kappa)$ we can define an equivalent probability measure $\mathbb{Q} \sim \mathbb{P}$ by

$$
\frac{d \mathbb{Q}}{d \mathbb{P}} \mid \mathcal{F}_{t}=M(t), \quad 0 \leq t \leq T .
$$

Under $\mathbb{Q}$ the processes

$$
\begin{aligned}
W^{\mathbb{Q}}(t) & =W(t)-\int_{0}^{t} \psi(s) d s, \quad 0 \leq t \leq T, \\
\tilde{N}^{\mathbb{Q}}(t, A) & =N(t, A)-\int_{0}^{t} \int_{A}(1+\kappa(s, v)) Q(s, d v) \eta(s) d s, \quad 0 \leq t \leq T,
\end{aligned}
$$

are, respectively, a $\mathbb{Q}$-Brownian motion and a $\mathbb{Q}$-compensated random measure. This important statement follows from the Girsanov-Meyer theorem (see Theorem III.40 in Protter (2004)).

Acknowledgments. This research was supported by the Foundation for Polish Science.

\section{References}

S. Ankirchner and P. Imkeller (2008), Quadratic hedging of weather and catastrophe risk by using short term climate prediction, preprint.

$\square$ S. Ankirchner, P. Imkeller and G. Dos Reis (2010), Pricing and hedging derivatives based on nontradable underlyings, Math. Finance 20, 289-312.

D. Becherer (2006), Bounded solutions to BSDE's with jumps for utility optimization and indifference hedging, Ann. Appl. Probab. 16, 2027-2054.

T. Bielecki, M. Jeanblanc and M. Rutkowski (2004), Hedging of defaultable claims, in: R. Carmona et al. (eds.), Paris-Princeton Lectures on Mathematical Finance 2003, Springer, 1-132.

[C. Blanchet-Scalliet and M. Jeanblanc (2004), Hazard rate for credit risk and hedging defaultable contingent claims, Finance Stoch. 8, 145-159.

M. Dahl, M. Melchior and T. Møller (2008), On systematic mortality risk and riskminimization with survival swaps, Scand. Actuarial J. 2, 114-146.

$\square$ M. Dahl and T. Møller (2006), Valuation and hedging of life isurance liabilities with systematic mortality risk, Insurance Math. Econom. 39, 193-217.

Ł. Delong (2010), An optimal investment strategy for a stream of liabilities generated by a step process in a financial market driven by a Lévy process, ibid. 47, 278-293.

European Commission (2010), Fifth Quantitative Impact Study: Call for Advice and Technical Specifications, http://ec.europa.eu/internal_market/insurance/solvency/ index_en.htm 
C. Frei and M. Schweizer (2009), Exponential utility indifference valuation in a general semimartingale model, in: Optimality and Risk-Modern Trends in Mathematical Finance. The Kabanov Festschrift, F. Delbaen et al. (eds.), Springer, 49-86.

M. Gundlach and F. Lehrbass (2004), CreditRisk+ in the Banking Industry, Springer, Berlin.

S. He, J. Wang and J. Yan (1992), Semimartingale Theory and Stochastic Calculus, CRC Press.

Y. Hu, P. Imkeller and M. Müller (2005), Utility maximization in incomplete markets, Annals Appl. Probab. 15, 1691-1712.

N. Kazamaki (1994), Continuous Exponential Martingales and BMO, Springer.

$\square$ Z. Liang, K. C. Yuen and J. Guo (2011), Optimal proportional reinsurance and investment in a stock market with Ornstein-Uhlenbeck process, Insurance Math. Econom. 49, 207215.

$\checkmark$ M. Ludkovski and V. R. Young (2008), Indifference pricing of pure endowments and life annuities under stochastic hazard and interest rates, ibid. 42, 14-30.

M. Mania and M. Schweizer (2005), Dynamic exponential utility indifference valuation, Ann. Appl. Probab. 15, 2113-2143.

H. Markowitz (1952), Portfolio selection, J. Finance 7, 77-91.

R. Merton (1969), Lifetime portfolio selection under uncertainty: the continuous time case, Rev. Econom. Statist. 1, 247-257.

$\square$ R. S. Perera (2010), Optimal consumption, investment and insurance with insurable risk for an investor in a Lévy market, Insurance Math. Econom. 46, 479-484.

P. Protter (2004), Stochastic Integration and Differential Equations, Springer.

$\square$ N. Wang (2007), Optimal investment for an insurer with exponential utility preference, Insurance Math. Econom. 40, 77-84.

$\square$ Z. Wang, J. Xia and L. Zhang (2007), Optimal investment for an insurer: The martingale approach, ibid. 40, 322-334.

Łukasz Delong

Institute of Econometrics, Division of Probabilistic Methods

Warsaw School of Economics

Al. Niepodległości 162

02-554 Warszawa, Poland

E-mail: lukasz.delong@sgh.waw.pl

Received on 5.9.2011;

revised version on 2.1.2012 
